\title{
GPPS-BJ-2019-162
}

\section{INVESTIGATION OF NUMERICAL ANALYSIS OF BLADE ROW INTERACTION UNSTEADY FLOWS WITHIN MULTI-ROW TURBOMACHINES USING THE TIME SPECTRAL METHOD}

\author{
Sen Zhang \\ Zhangsen1998@mail.nwpu.edu.cn \\ School of Power and Energy, Northwestern \\ Polytechnical University, Xi'an, Shaanxi, 710072, \\ China
}

\author{
Dingxi Wang \\ dingxi_wang@nwpu.edu.cn \\ Shaanxi Key Laboratory of Internal Aerodynamics \\ in Aero-engines, Northwestern Polytechnical \\ University, 127, Youyixi Road, Shaanxi, 710072,
}

\author{
Xiuquan Huang \\ xiuquan_huang@nwpu.edu.cn \\ Shaanxi Key Laboratory of Internal Aerodynamics \\ in Aero-engines, Northwestern Polytechnical \\ University, 127, Youyixi Road, Shaanxi, 710072, \\ China
}

\begin{abstract}
This paper presents a method for selecting harmonics and spinning modes for a rotor-stator interaction analysis using the time spectral method. Discrete Fourier transform of the circumferential distribution of conservative variables is performed to provide the necessary information for guiding selection of harmonics. The rules for the spinning mode selection are summarized from a lot of numerical analyses by changing the numbers of spinning modes. NASA stage 35 was used to demonstrate the effectiveness of the proposed selection method.
\end{abstract}

\section{INTRODUCTION}

Turbomachine is an integral part of an aeroengine or industrial gas turbine. To have a first-class aeroengine means to design an efficient turbomachine. The road to get a good turbomachine is bumpy because the flow inside turbomachines is always unsteady. It is much more difficult to comprehend the mechanism of unsteady flow than to know the existence of it. To better understand unsteady phenomenon, many numerical methods have been developed to analyse unsteady flows. The most direct method to analyse unsteady flow field is the time domain time marching method, which is relatively simple to implement. But the main downside is that it involves large memory and requires long computational time. In order to save computational time, we need a feasible path to analyse unsteady flow. The unsteady flow is sometimes periodic especially when it stems from a rotor-stator interaction. Therefore, the unsteady flow field can be approximated with truncated Fourier series. Besides, the blades in one row are identical to each other and evenly spaced along the circumferential direction. The unsteady flows in different passages have the same amplitude and a fixed phase difference. All these conditions are favourable for development of reduced order methods. There are several reduced order methods being proposed: the linear harmonic method [1 3], the nonlinear harmonic method [4 5], and the time spectral method [6 12]. In this investigation, we choose the time spectral method as the reduced order method to be investigated.

The unsteady flow analysis based upon the time spectral method resolves unsteady flow at some selected time instants, which are quite often different for different blade rows. This is because in different blade rows, different number of time instants, due to different number of harmonics, are used. The selection of the number of harmonics is essential for the time spectral method. If the number of harmonics is too few, the flow field information cannot be obtained accurately. However, if the number of harmonics is too many, it will consume too much CPU time, leading to bad stiffness issue and even solution instability. Therefore, choosing a proper number of harmonics is very imperative in a time spectral analysis. Raymond C. Maple et al. [13] has proposed an adaptive harmonic balance method to determine the number of harmonics, which is difficult to implement unfortunately. Gomar et al. [14] has proposed a method of extracting tangential variations of the axial 
momentum to determine the number of harmonics. However, the number of harmonics predicted through this method is the same for both upstream and downstream blade row. According to some previous study [15], the wake from an upstream blade row is harder to resolve than the pressure field of a downstream blade row. To perform an analysis economically, less harmonics could be used in an upstream blade row than that in a downstream blade row. Huang and Wang [16] perform spectral analysis on the circumferential distribution of steady flow field to choose harmonics. Entropy from an upstream blade row is used to determine the number of harmonics needed for the downstream domain and the static pressure from a downstream blade row is used to determine the number of harmonics needed for the upstream domain. But the wake usually includes entropy waves and vorticity waves, it is not enough to just take the entropy waves into consideration. A different approach is needed to analyse the wake more thoroughly. In this paper, a method based upon Discrete Fourier transform is introduced to determine the number of harmonics at the exit of an upstream blade row and the inlet of a downstream row respectively.

When the number of harmonics is determined, how to match spinning modes properly is the next important step to obtain accurate results because spinning modes [17] which are generated in a rotor-stator interaction are blade-row coupling entities. The time and space mode decomposition and matching method [15] has provided a complete method for matching those spinning modes across a rotor-stator interface. Good approximation of the flow field can be achieved through a few numbers of spinning modes. This paper will investigate how spinning modes affect the time averaged and unsteady components of the flow field under consideration.

\section{METHODOLOGY}

In order to obtain the minimum number of harmonics used in a time spectral analysis, discrete Fourier transform of the circumferential distribution of conservative variables of a steady flow field is performed at the exit of an upstream row and the inlet of a downstream row. We know that the unsteady disturbances between blade rows are mainly potential disturbances and wake disturbances. The wake disturbances consist of entropy and vorticity disturbances. The potential disturbances consist of pressure disturbances. The pressure, entropy and vorticity are calculated through solving the Euler equations or Navier-Stokes equations. Therefore, if the information represented by the conservative variables can be retained with appropriate number of harmonics, the unsteady disturbances can also be obtained. The Navier-Stokes equations with the Spalart-Allmaras turbulence model [18] include six conservative variables in total: $\rho, \rho u, \rho v, \rho w, \rho E, \rho \lambda$.

In order to clarify this selection process, an example will be given to illustrate it. The flow field along the circumferential direction is periodic because of the periodic boundary condition. Therefore, only one passage is chosen to perform a steady analysis. Figure 1 shows the density circumferential distribution of one passage at $50 \%$ span of the rotor exit. At the exit of an upstream blade row and the inlet of a downstream blade row, discrete Fourier transform will be performed on the circumferential distribution of the conservative variables at a fixed span. When the discrete Fourier transform is done, we will have the amplitude of each harmonic like figure 2. Square each amplitude and add them up, the summation can be named 'flow energy'. If the summation of the square of the amplitude from the first harmonic to the nth harmonic accounts for $99 \%$ of the 'flow energy', then the number of harmonics required for analysis is $\mathrm{n}$. The reason for choosing $99 \%$ as the criterion will be explained later. In figure 3, if exact $99 \%$ of 'flow energy' is contained, the accurate number of harmonics is 5.2681. But to ensure the harmonic number is an integer and retain at least $99 \%$ of the information, 6 harmonics are chosen. Using

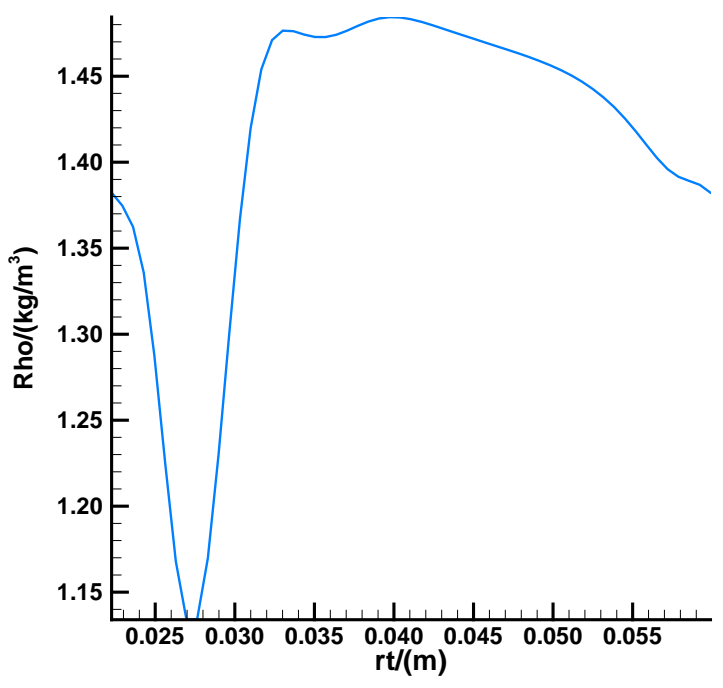

Figure 1 Density circumferential distribution at $\mathbf{5 0 \%}$ span of the rotor exit.

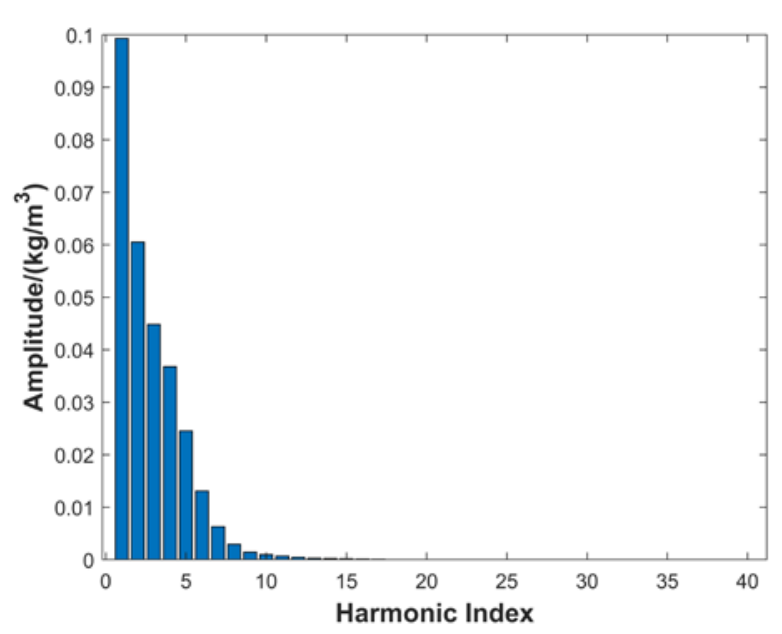

Figure 2 Amplitudes of each harmonic. 


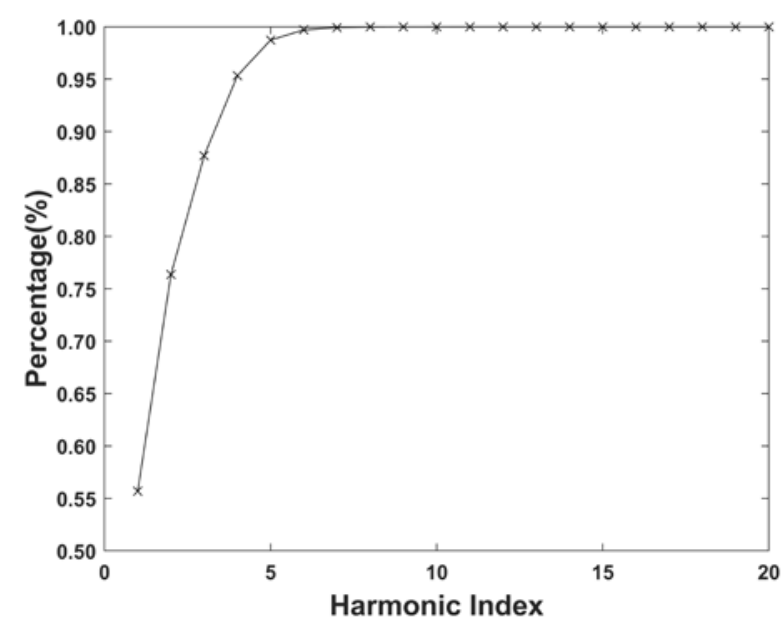

Figure 3 Percentage of 'flow energy' at corresponding harmonics.

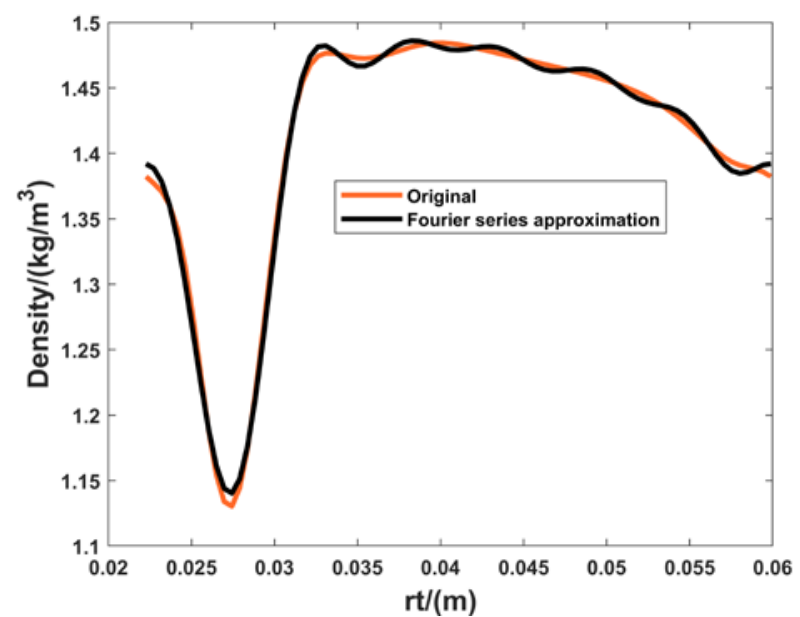

Figure 4 Comparison of the original density circumferential distribution and the Fourier series approximation distribution with 6 harmonics.

6 harmonics to approximate the original distribution, the approximation looks good in Figure 4. Besides, nine spans are picked to see the change of the number of harmonics needed along the blade. Based on the fact that the perturbation to $\rho \lambda$ only propagates downstream, $\rho \lambda$ will not be included to perform spectral analysis at the inlet of a downstream blade row.

Another part of work is to find out the effect of spinning modes on the time averaged and unsteady component of the flow field under consideration. It is based on the time and space mode decomposition and matching method [15]. The time and space mode decomposition and matching method divided the spinning modes into five groups:

The first group contains the matching of time averaged circumferentially uniform flow field. There is only one pair of mode.

The second group is the matching of time averaged circumferentially nonuniform flow field in the rotor domain and unsteady flow field in the stator domain. Here are $n_{2}$ pairs.

$$
\left(0, j \times N_{1}\right) \leftrightarrow\left(j \times N_{1} \times \omega, j \times N_{1}\right)
$$

where $j \in\left[1, n_{2}\right]$. Each bracket in the above expression denotes one row. The first part in one bracket is time mode, the second part is spatial mode.

The third group is the matching of time averaged circumferentially nonuniform flow field in the stator domain and unsteady flow field in the rotor domain. Here are $n_{1}$ pairs.

$$
\left(i \times N_{2} \times \omega,-i \times N_{2}\right) \leftrightarrow\left(0, i \times N_{2}\right)
$$

where $i \in\left[1, n_{1}\right]$.

The fourth group is the matching of unsteady flow field in the rotor domain and unsteady flow field in the stator domain. Here are $n_{1} \times n_{2}$ pairs.

$$
\text { where } i \in\left[1, n_{1}\right] \text { and } j \in\left[1, n_{2}\right] \text {. }
$$

The fifth group is also the matching of unsteady flow field in the rotor domain and unsteady flow field in the stator domain. But the spatial mode of the fifth group is different from that of the fourth group. Here are $n_{1} \times n_{2}$ pairs.

$$
\text { where } i \in\left[1, n_{1}\right] \text { and } j \in\left[1, n_{2}\right] \text {. }
$$

The total number of spinning modes is given by the following formula

$$
1+n_{2}+n_{1}+n_{1} \times n_{2} \times 2
$$

The first three groups can provide some basic information about the flow filed, which contain $1+n_{1}+n_{2}$ spinning modes. The aim of this paper is to see how the fourth and the fifth group affect the flow field. Therefore, the selection will start from $1+n_{1}+n_{2}$ spinning modes. Increase the spinning modes step by step to determine the proper number of spinning modes with which the flow field can be well approximated.

\section{THE SELECTION OF THE NUMBER OF HARMONICS}

The transonic compressor NASA stage 35 [19] is used as the test case which has 36 rotor blades and 46 stator blades. In this case, the upstream blade row is a rotor and the downstream blade row is a stator. One blade passage for each blade row is included in the computational domain. The mesh for the upstream blade row domain consists of 57 grid points in the circumferential direction, 73 grid points in the spanwise direction and 225 grid points in the streamwise direction. The mesh for the downstream blade row domain consists of 57 grid points in the circumferential direction, 73 grid points in the spanwise direction and 217 grid points in the streamwise direction. Figure 5 shows the blade to blade view and the meridional view of the NASA stage 35 grid.

For the boundary condition setting, radial distribution of total pressure, total temperature, flow angles are applied to the inlet and radial profile of static pressure is specified at the outlet. The phase-shift boundary condition is used at geometric periodic boundaries. For the solid wall boundary condition, the slip wall condition with the wall function is utilized. The time and space decomposition and matching method is adopted in the rotor-stator coupling. As for the time domain analysis, there are 100 physical time steps in one rotor blade passing period. 20 internal steps are included in one physical time step.

The operational condition of case analysed here is near the design point. To find out the number of harmonics 
needed to retain 99\% of 'flow energy', conservative flow variables at nine spans have been chosen for the selection. Table 1 shows that the numbers of harmonics needed at different spans for different conservative flow variables. In the first column, take ' $50 \%$ out' as an example, '50\%' means the circumferential distribution at $50 \%$ span is chosen, 'out' denotes that the analysis is performed at the rotor exit. Similarly, 'in' denotes it is at the stator inlet. 'Max' denotes
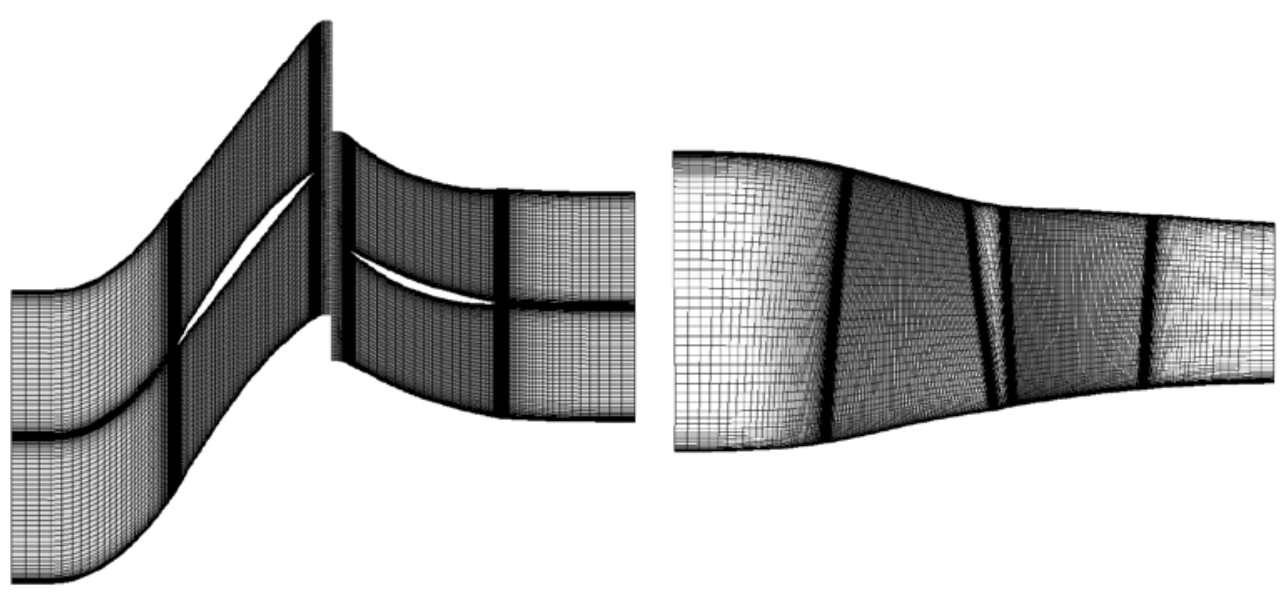

Figure 5 Blade to blade view and meridional view of the grid.

Table 1 The number of harmonics at different spans

\begin{tabular}{|c|c|c|c|c|c|c|c|}
\hline Span & $\rho \lambda$ & $\rho$ & $\rho u$ & $\rho v$ & $\rho w$ & $\rho E$ & Max \\
\hline 5\%_out & 5 & 6 & 5 & 5 & 7 & 6 & 7 \\
\hline 10\%_out & 6 & 5 & 5 & 5 & 10 & 5 & 10 \\
\hline 20\%_out & 7 & 6 & 6 & 6 & 10 & 6 & 10 \\
\hline 40\%_out & 6 & 6 & 6 & 5 & 7 & 6 & 7 \\
\hline 50\%_out & 5 & 6 & 5 & 5 & 7 & 5 & 7 \\
\hline 60\%_out & 5 & 5 & 5 & 4 & 6 & 4 & 6 \\
\hline 80\%_out & 5 & 5 & 5 & 5 & 6 & 4 & 6 \\
\hline 90\%_out & 5 & 5 & 5 & 5 & 6 & 5 & 6 \\
\hline 95\%_out & 6 & 5 & 5 & 5 & 6 & 4 & 6 \\
\hline 5\%_in & - & 3 & 3 & 3 & 3 & 3 & 3 \\
\hline 10\%_in & - & 3 & 3 & 3 & 3 & 3 & 3 \\
\hline 20\%_in & - & 2 & 2 & 2 & 2 & 2 & 2 \\
\hline 40\%_in & - & 2 & 2 & 2 & 2 & 2 & 2 \\
\hline 50\%_in & - & 2 & 2 & 2 & 2 & 2 & 2 \\
\hline 60\%_in & - & 2 & 2 & 2 & 2 & 1 & 2 \\
\hline 80\%_in & - & 2 & 2 & 2 & 2 & 2 & 2 \\
\hline 90\%_in & - & 2 & 2 & 2 & 2 & 2 & 2 \\
\hline 95\%_in & - & 2 & 2 & 2 & 2 & 2 & 2 \\
\hline
\end{tabular}

the maximum number of harmonics needed for conservative variables at that exit or inlet. The following two points can be drawn from the data presented in the table:

1. The number of harmonics used to analyse the flow field at the exit of the rotor is about 6 or 7, at the inlet of the stator is about 2 or 3 . The overall distribution is relatively uniform at different spans along the blade, although there is a need of more harmonics at the lower span.
2. $\rho w$ needs more harmonics to resolve than other conservative variables.

Because these three variables have the same dimension, comparison between $\rho u, \rho v, \rho w$ is made to investigate why more harmonics are needed to fit $\rho \mathrm{w}$. Before that, a brief review of temporal Fourier series will be provided. The temporal Fourier series generally have a time averaged part and sinusoidal parts. Whether the fitness is good or not, it totally depends on these two parts. When the time averaged 
value is given, the basic horizon line is determined. To approximate the original curve, some sinusoidal functions will be added to that horizon line. The more sinusoidal functions are counted, the better the approximation is. However, the first few sinusoidal functions with large amplitudes are the ones that make the biggest difference. Those sinusoidal functions with small amplitudes will flatten out the wiggly parts but will not alter the general shape of the original curve.

Following the above illustration, detailed data of time averaged part and the first ten harmonic amplitudes at $10 \%$ span of the rotor exit have been extracted, shown in Table 2. The percentage of amplitude of each harmonic to time averaged value is listed in Table 3. All data in Table 2 have the same units $\mathrm{kg} /\left(\mathrm{m}^{2} \cdot \mathrm{s}\right)$.

Table 2 Time averaged value and amplitudes of the first ten harmonics

\begin{tabular}{cccc}
\hline \hline & $\rho u$ & $\rho v$ & $\rho w$ \\
\hline Time average & 283.99 & 277.72 & 32.80 \\
First order & 39.02 & 49.39 & 7.64 \\
Second order & 29.82 & 37.90 & 6.31 \\
Third order & 21.49 & 24.72 & 6.55 \\
Fourth order & 13.61 & 14.41 & 6.13 \\
Fifth order & 6.94 & 7.92 & 5.16 \\
Sixth order & 3.18 & 4.59 & 4.23 \\
Seventh order & 1.59 & 2.79 & 3.40 \\
Eighth order & 0.80 & 1.62 & 2.66 \\
Ninth order & 0.52 & 0.93 & 2.00 \\
Tenth order & 0.44 & 0.55 & 1.45 \\
\hline \hline
\end{tabular}

Table 3 Percentage of each harmonic amplitude to time averaged value

\begin{tabular}{cccc}
\hline \hline & $\rho u$ & $\rho v$ & $\rho w$ \\
\hline First order & $13.74 \%$ & $17.79 \%$ & $23.30 \%$ \\
Second order & $10.50 \%$ & $13.65 \%$ & $19.25 \%$ \\
Third order & $7.57 \%$ & $8.90 \%$ & $19.98 \%$ \\
Fourth order & $4.79 \%$ & $5.19 \%$ & $18.69 \%$ \\
Fifth order & $2.44 \%$ & $2.85 \%$ & $15.73 \%$ \\
Sixth order & $1.12 \%$ & $1.65 \%$ & $12.88 \%$ \\
Seventh order & $0.56 \%$ & $1.00 \%$ & $10.38 \%$ \\
Eighth order & $0.28 \%$ & $0.58 \%$ & $8.10 \%$ \\
Nine order & $0.18 \%$ & $0.34 \%$ & $6.10 \%$ \\
Tenth order & $0.16 \%$ & $0.20 \%$ & $4.42 \%$ \\
\hline \hline
\end{tabular}

The reason for choosing data at $10 \%$ span of the rotor exit is that $\rho w$ apparently needs more harmonics at $10 \%$ span than that at other spans. From Table 2, we could know that the percentage of each amplitude to the time averaged value is still relatively large even in the tenth harmonic of $\rho w$, which means these amplitudes are necessary to approximate the original distribution of $\rho w$. But the time averaged part of $\rho w$ is apparently much smaller than other two counterparts. Moreover, the amplitudes of first five harmonics of $\rho w$ is also smaller than that of $\rho u, \rho v$. In this situation, it is not worth choosing so many harmonics to approximate the distribution of $\rho w$, which has a small magnitude. This is applicable at other spans. Therefore, after thoughtful consideration, we decide to exclude $\rho w$ in the selection of the number of harmonics.

In order to select a proper number of harmonics conveniently, it is not necessary to analyse data at so many spans. Without loss of generality, we could choose three spans 10\%, 50\% 90\% for the selection (see Table 4). Average the numbers at three spans of the rotor exit to decide the number of harmonics used in a stator domain. In this case, it is 6 . In the same way, the number of harmonics used in rotor domain is 3 .

Table 4 The number of harmonics at $10 \%, 50 \%, 90 \%$ span

\begin{tabular}{ccccccc}
\hline \hline Span & $\rho \lambda$ & $\rho$ & $\rho u$ & $\rho v$ & $\rho E$ & Max \\
\hline 10\%_out & 6 & 5 & 5 & 5 & 5 & 6 \\
50\%_out & 5 & 6 & 5 & 5 & 5 & 6 \\
90\%_out & 5 & 5 & 5 & 5 & 5 & 5 \\
10\%_in & - & 3 & 2 & 2 & 2 & 3 \\
50\%_in & - & 2 & 2 & 2 & 2 & 2 \\
90\%_in & - & 2 & 2 & 3 & 2 \\
\hline \hline
\end{tabular}

Figure 6 shows the contour of entropy distribution at $50 \%$ span. Contours at the top left and top right and bottom left are calculated through the time spectral method, the bottom right one is from a time domain solution. The first number in the bracket denotes the number of harmonics used in the rotor domain, the second number denotes the number of harmonics used in the stator domain. When the number of harmonics is just two for both rows, it is not enough and the flow field cannot be well resolved. With four harmonics for both the upstream and downstream domains, the wake can be resolved better than the solution with two harmonics. When the number of harmonics is 3 for the rotor domain and 6 for the stator domain, the entropy distribution looks good. The wake from the rotor domain is continuous across the interface. There is not a clear difference between the time spectral solution and time domain solution. Another two 
representative spans (10\% and $90 \%$ spans) have also been chosen to make a comparison like those in figure 7 and figure 8 respectively. To save space, we only compare the result of the time spectral method using 3 harmonics for the upstream domain and 6 harmonics for the downstream domain with the result of a time domain dual time stepping analysis. The difference between the time spectral method solution and time domain solution is also very small. These comparisons can demonstrate the effectiveness of the proposed method.

\section{entropy: $\quad 0 \quad 20 \quad 40 \quad 60 \quad 80100120140160180200220240260280$}

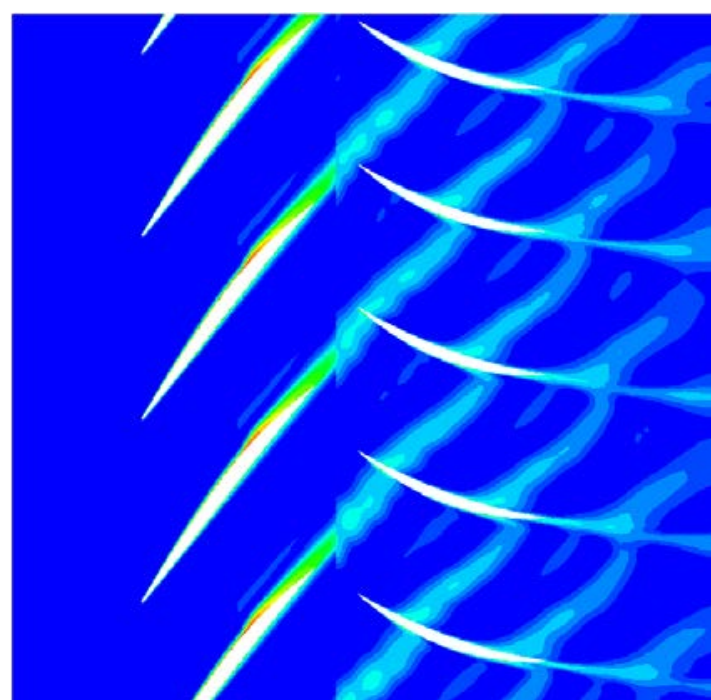

$(2-2)$

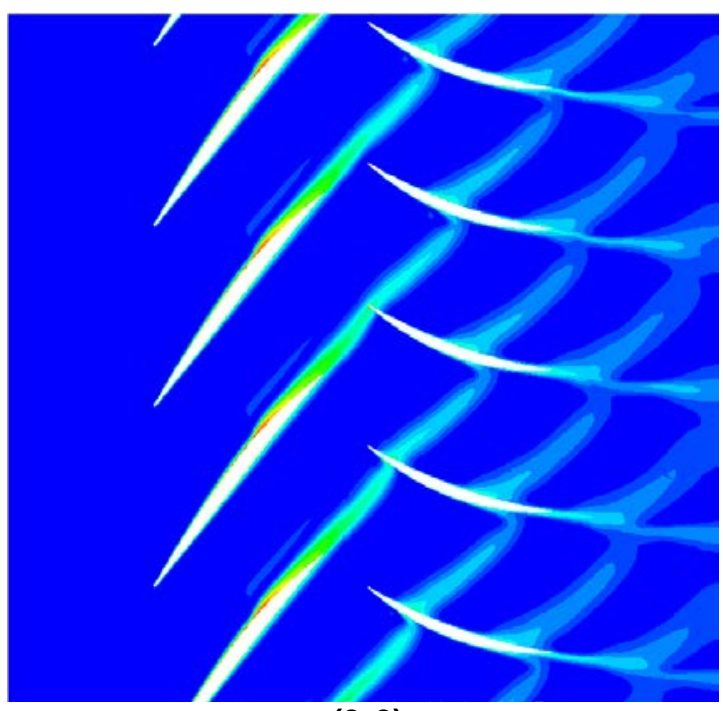

(3-6)

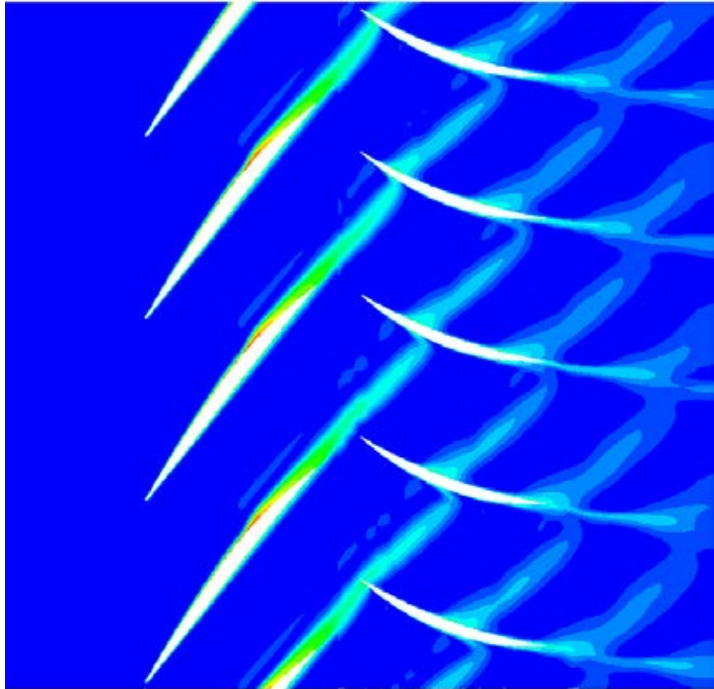

(4-4)

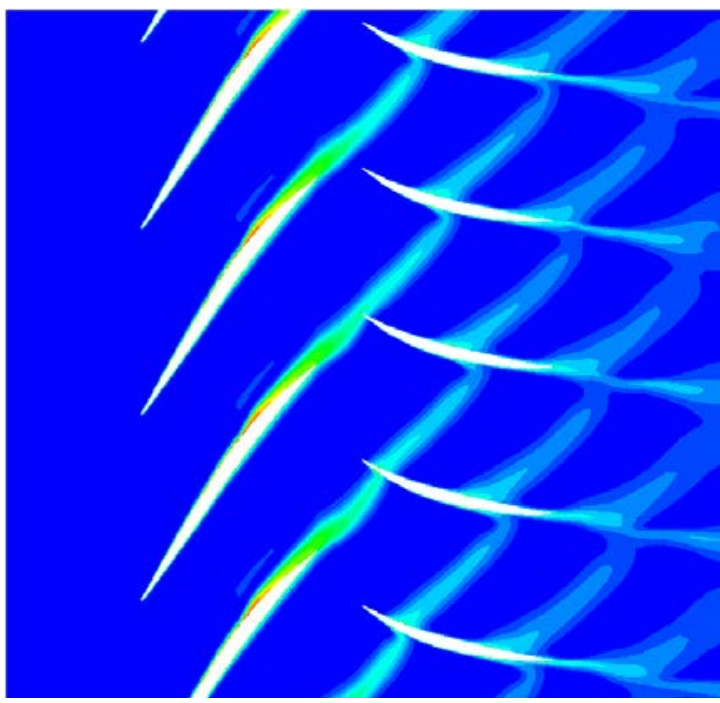

(Time Domain)

Figure 6 Comparison of entropy distribution using different harmonics at $50 \%$ span. 


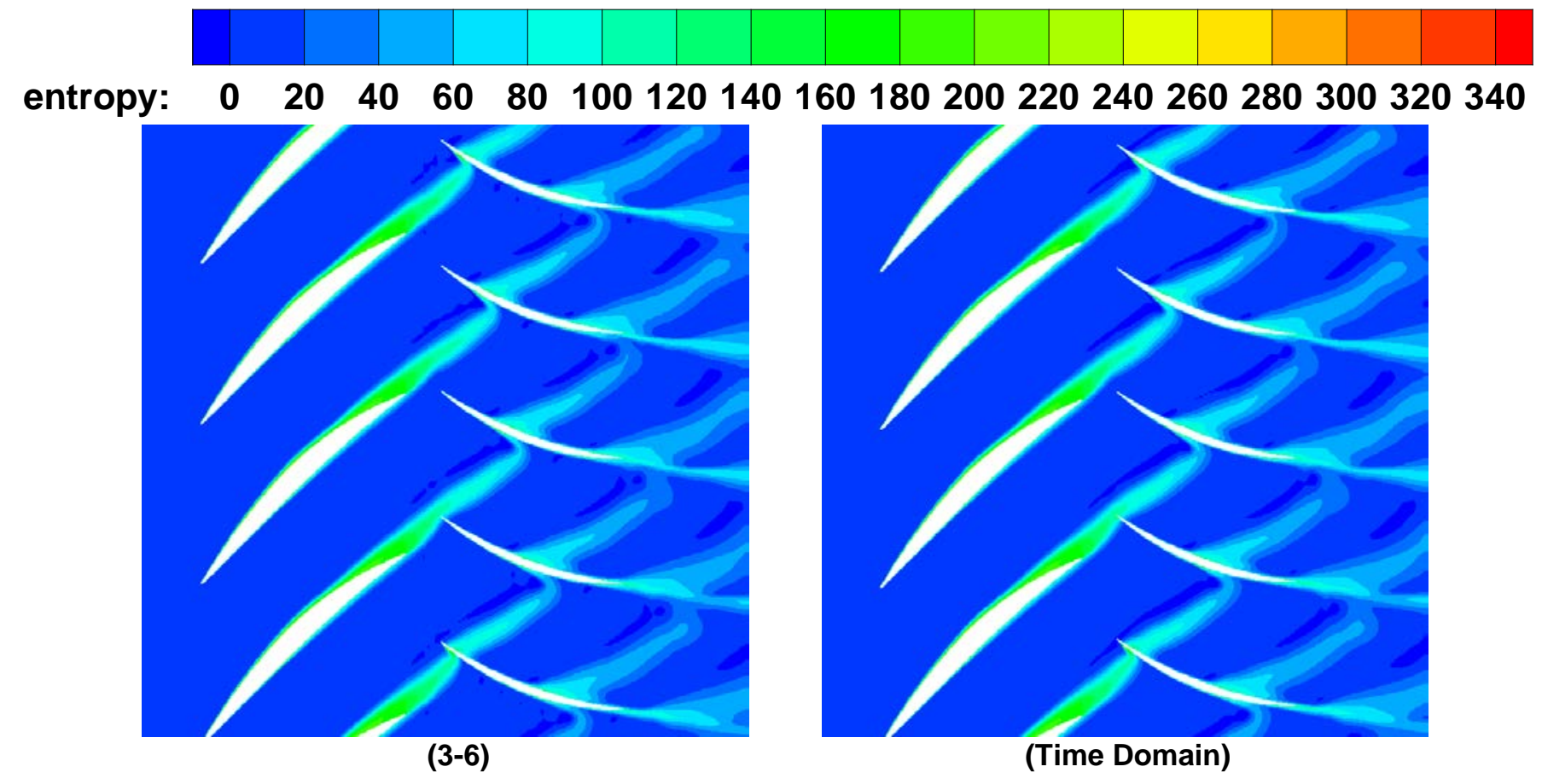

Figure 7 Comparison of entropy distribution at $10 \%$ span.

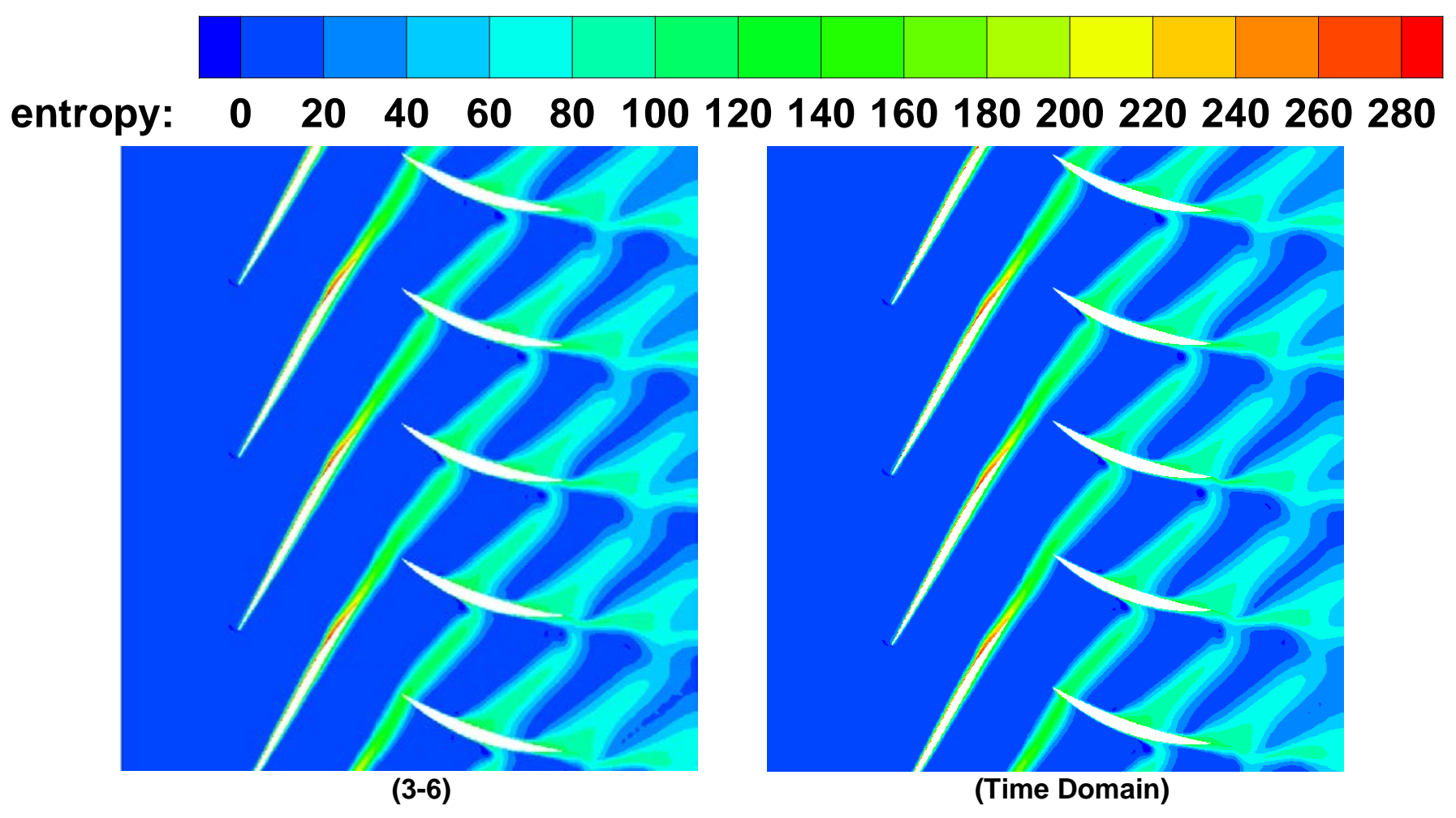

Figure 8 Comparison of entropy distribution at $90 \%$ span. 
Following the above procedure, the number of harmonics predicted will be different under different criterion. If $90 \%$ is chosen as the criterion, the number of harmonics will be 1-4 (The first number denotes the number of harmonics used for the rotor domain and the second number denotes the number of harmonics used for the stator domain). When 95\%, 99\%, 99.9\% are chosen as the criterion, the number of harmonics will be 2-5, 3-6, 4-8, respectively. Figure 9 shows the entropy distribution at 50\% span. Figure
10 shows the comparison of computational time when using different criterion. When the criterion is $90 \%$, it can be seen clearly that the flow field is not well resolved. Meanwhile, the flow field is still not good enough when setting 95\% as the criterion. There is no obvious difference for the flow field when the criterion is set to $99 \%, 99.9 \%$. The entropy distribution is continuous across the interface. However, it is too time consuming when choosing $99.9 \%$ as the criterion. Therefore, $99 \%$ is chosen to select the number of harmonics in this paper.

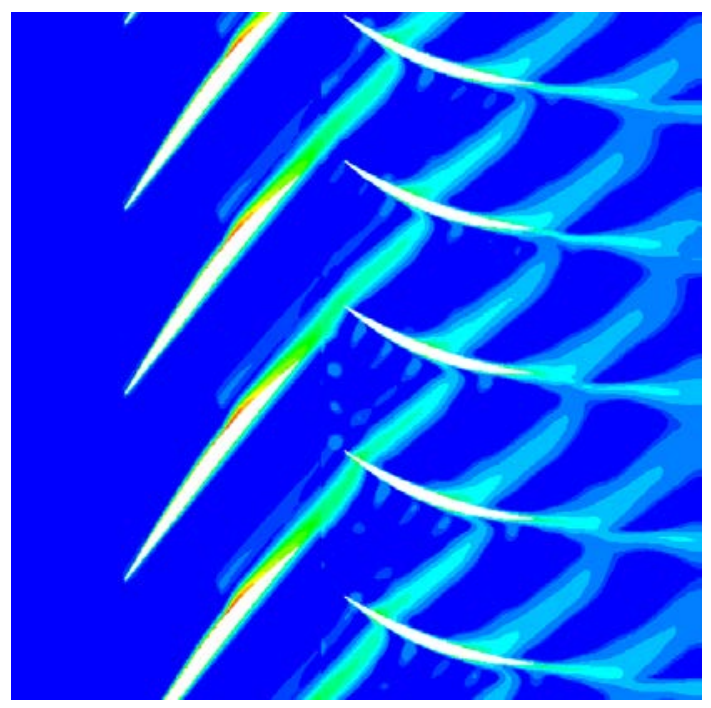

(90\%)

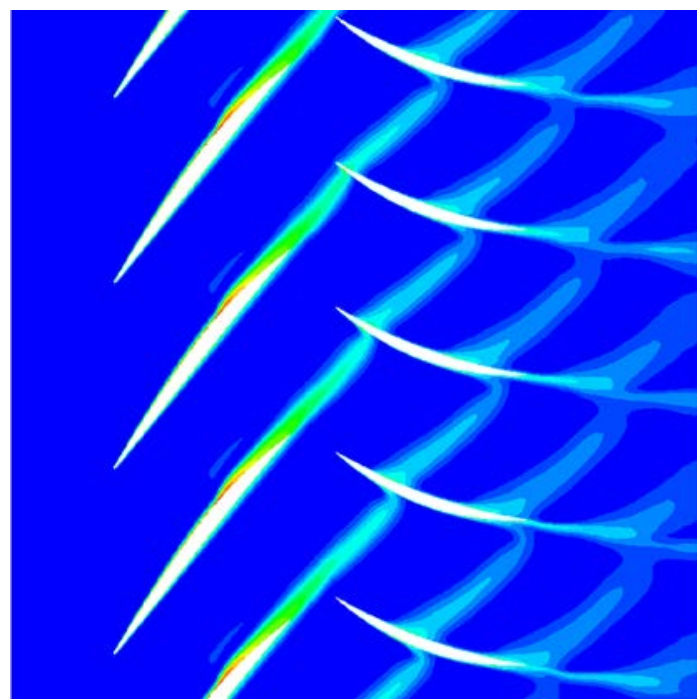

$(99 \%)$

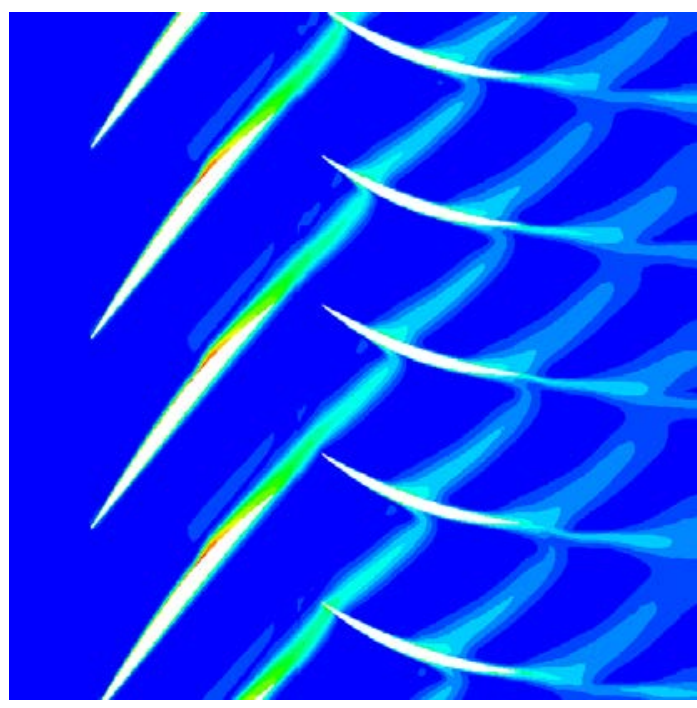

(95\%)

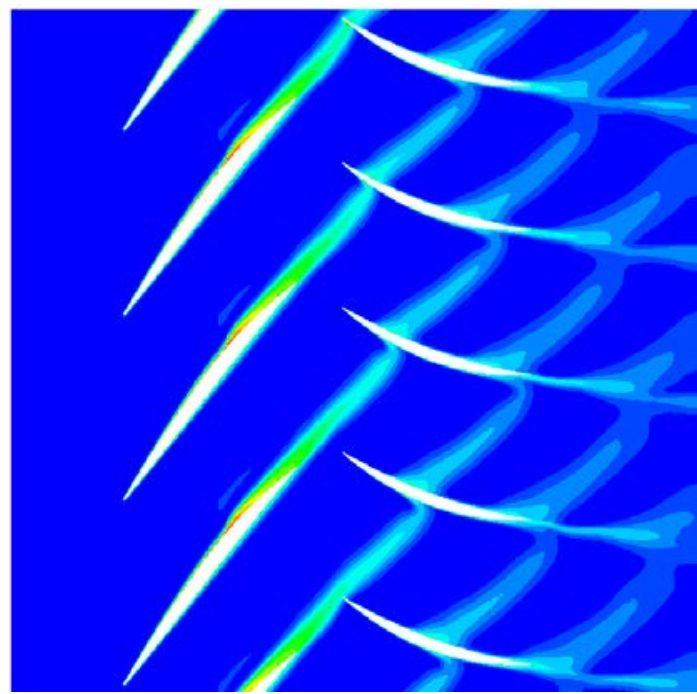

$(99.9 \%)$

Figure 9 Comparison of entropy distribution when choosing different criterion at $50 \%$ span. 


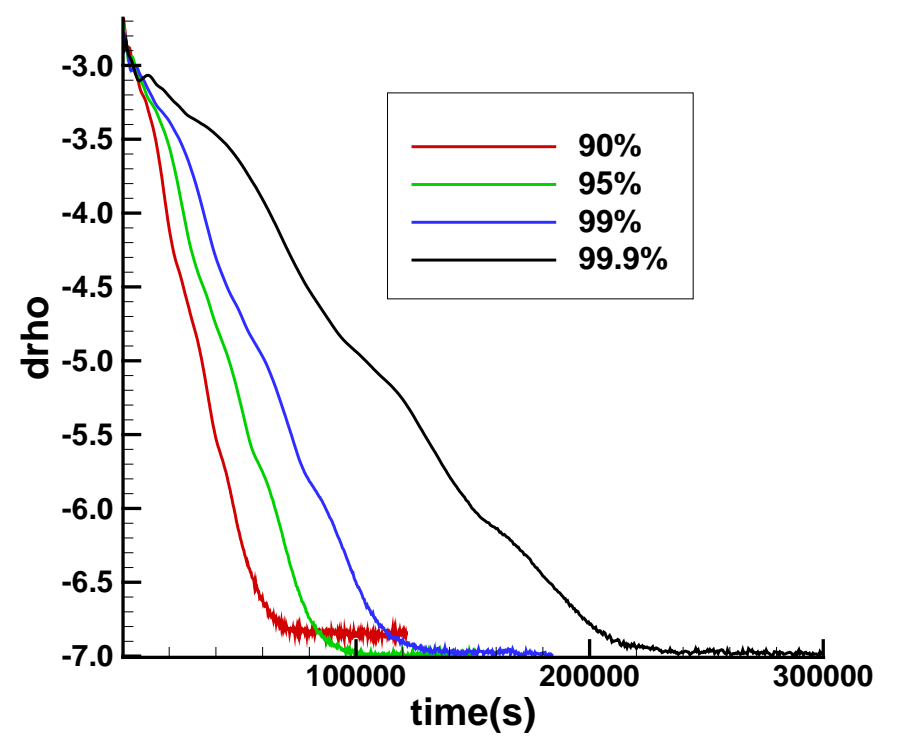

Figure 10 Comparison of computational time when choosing different criterion.

\section{THE SELECTION OF SPINNING MODES}

NASA stage 35 case with the same mesh is used in the selection of spinning modes. This analysis was performed with 3 harmonics for the upstream blade row and 6 harmonics for the downstream blade row based on the proposed harmonic selection method. According to the time and space mode decomposition and match method, there are $1+n_{1}+n_{2}+2 \times n_{1} \times n_{2}$ spinning modes for a case with $n_{1}$ harmonics for one blade row and $n_{2}$ harmonics for the other row. For this particular case, there are in total 46 pairs spinning modes in the analysis. Besides, the selection will start from $1+n_{1}+n_{2}$ pairs spinning modes, which is 10 pairs in this case.

There is not too much difference in the overall performance and time averaged static pressure between solution using the complete spinning modes and solution using reduced spinning modes (see Figure 11 and Figure 12). But when it comes to the amplitude of harmonic pressure, more spinning modes need to be included to get a good approximation for the amplitude of higher order harmonic pressure (see Figure 13, Figure 14 and Figure 15). Hence, if the focus is on the overall performance and time averaged static pressure, basic spinning modes are enough, which means the first three groups are enough.
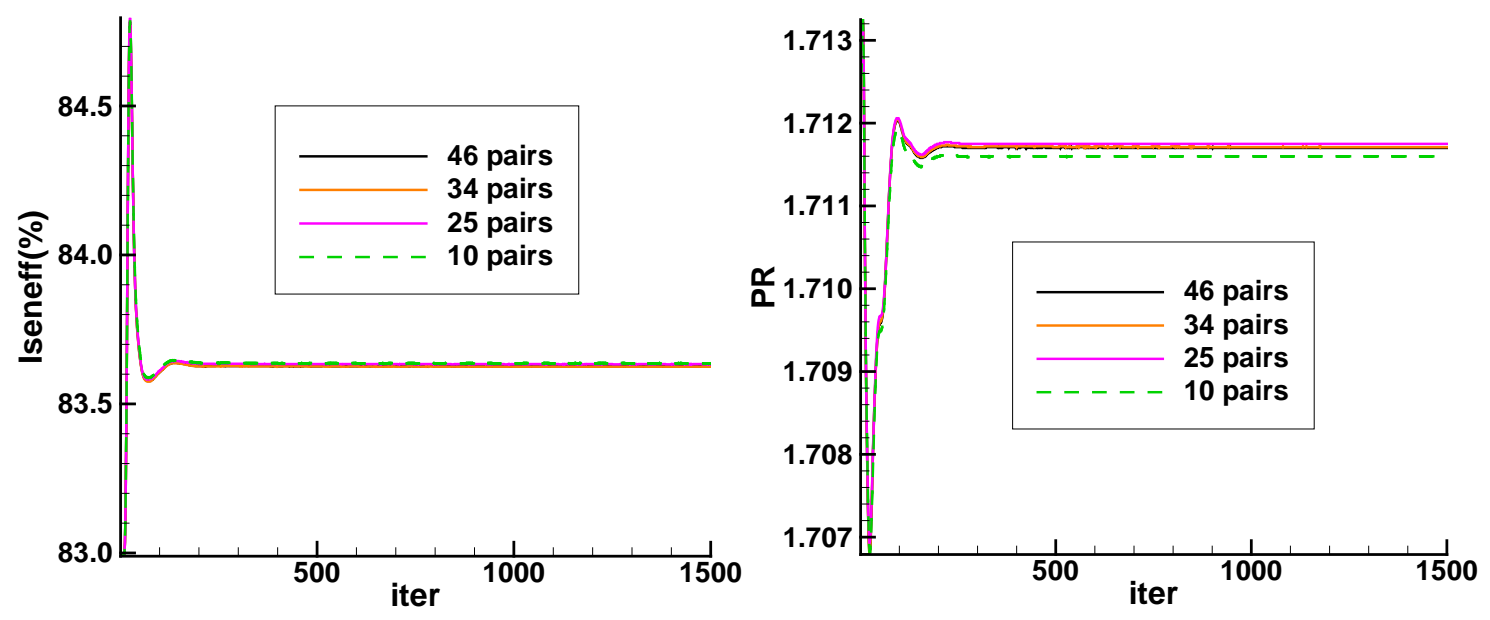

Figure 11 Comparison of the isentropic efficiency (left) and pressure ratio(right) between solution using the complete spinning modes and solution using reduced spinning modes. 

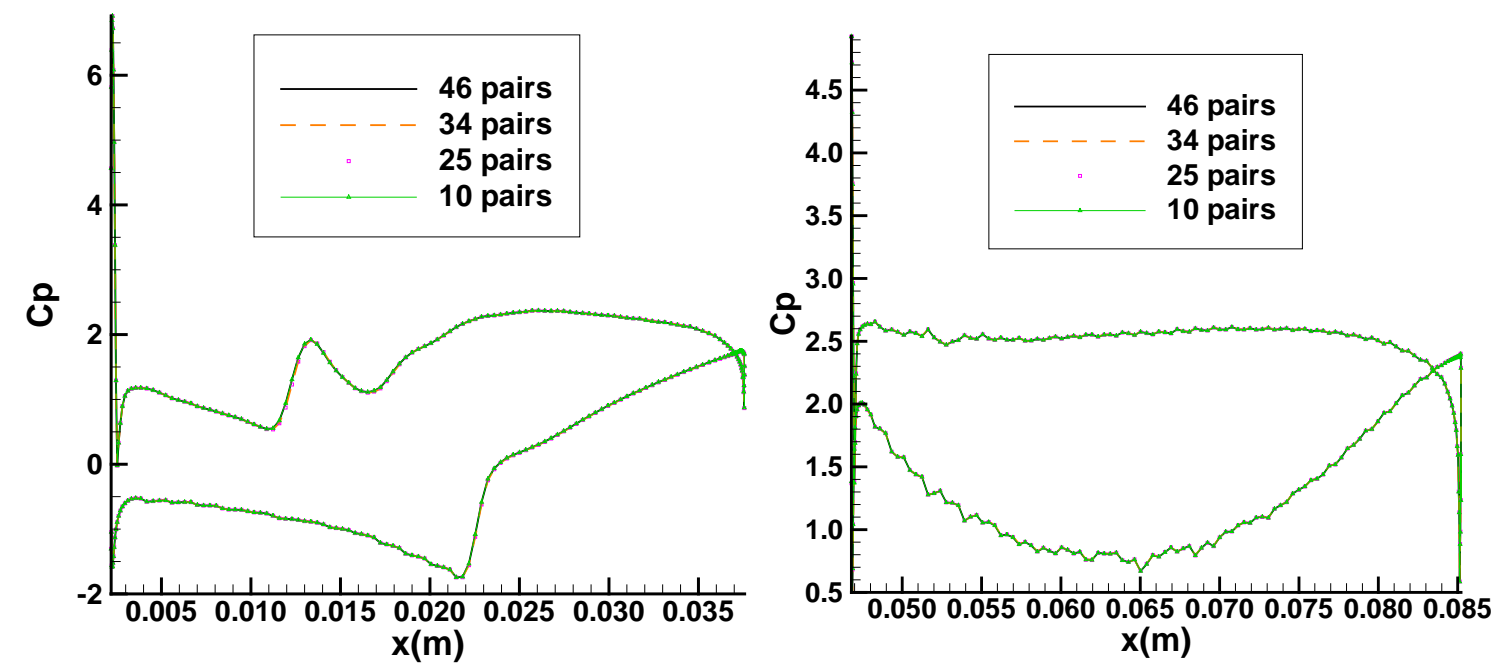

Figure 12 Comparison of the time averaged static pressure coefficient stream-wise distribution at $50 \%$ span of the rotor (left) and the stator (right).
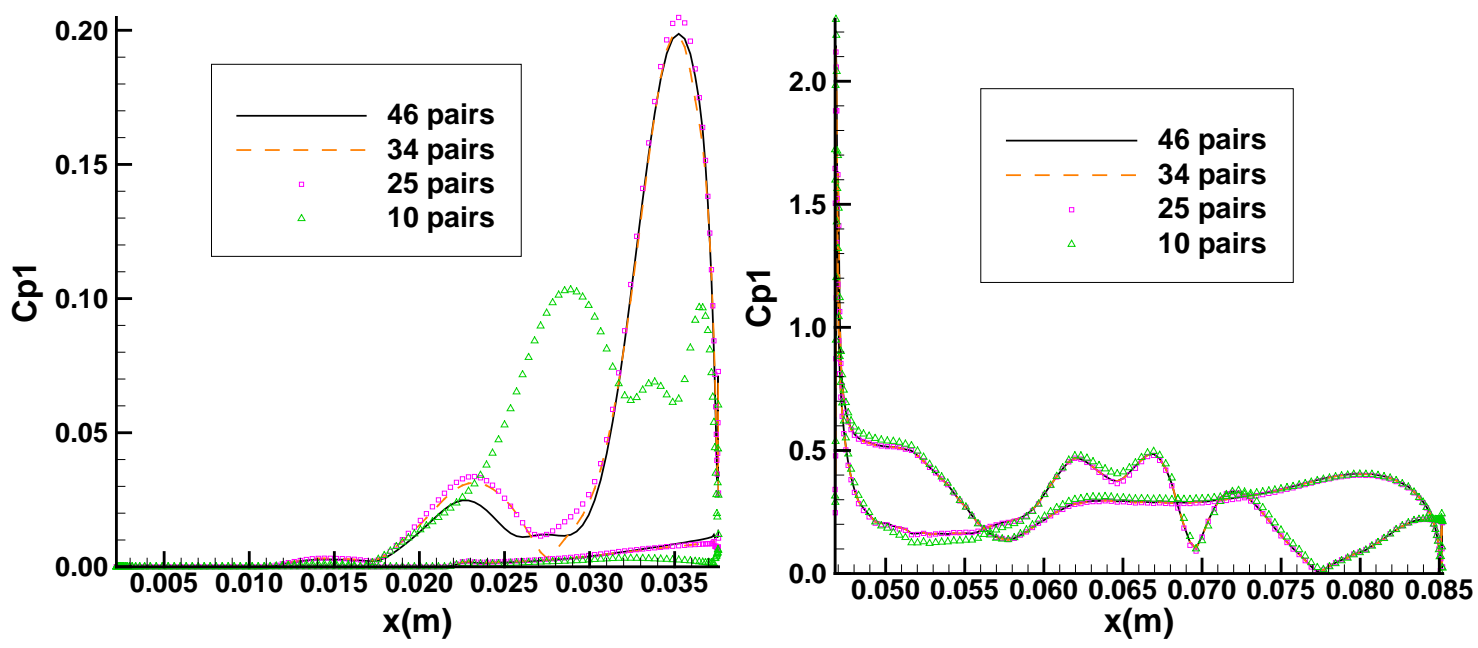

Figure 13 Comparison of amplitude of the first harmonic pressure coefficient stream-wise distribution at $50 \%$ span of the rotor (left) and the stator (right).
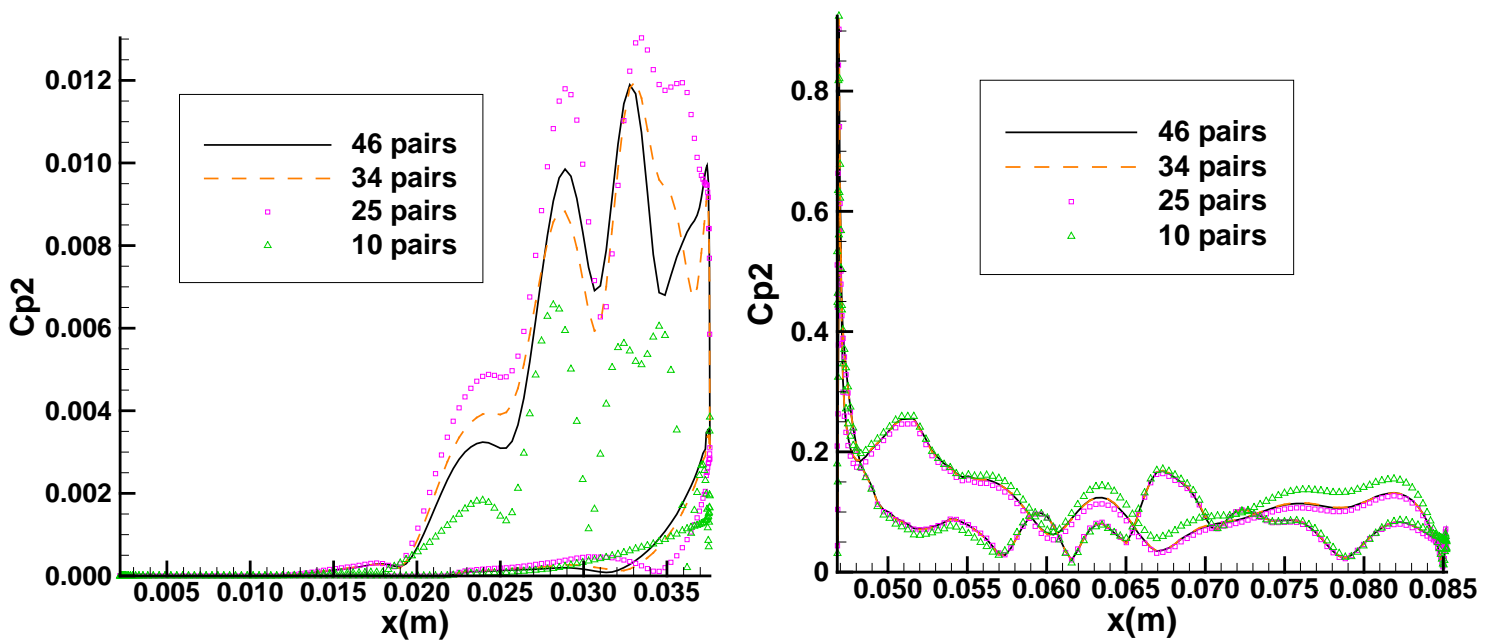

Figure 14 Comparison of amplitude of the second harmonic pressure coefficient stream-wise distribution at $50 \%$ span of the rotor (left) and the stator (right). 

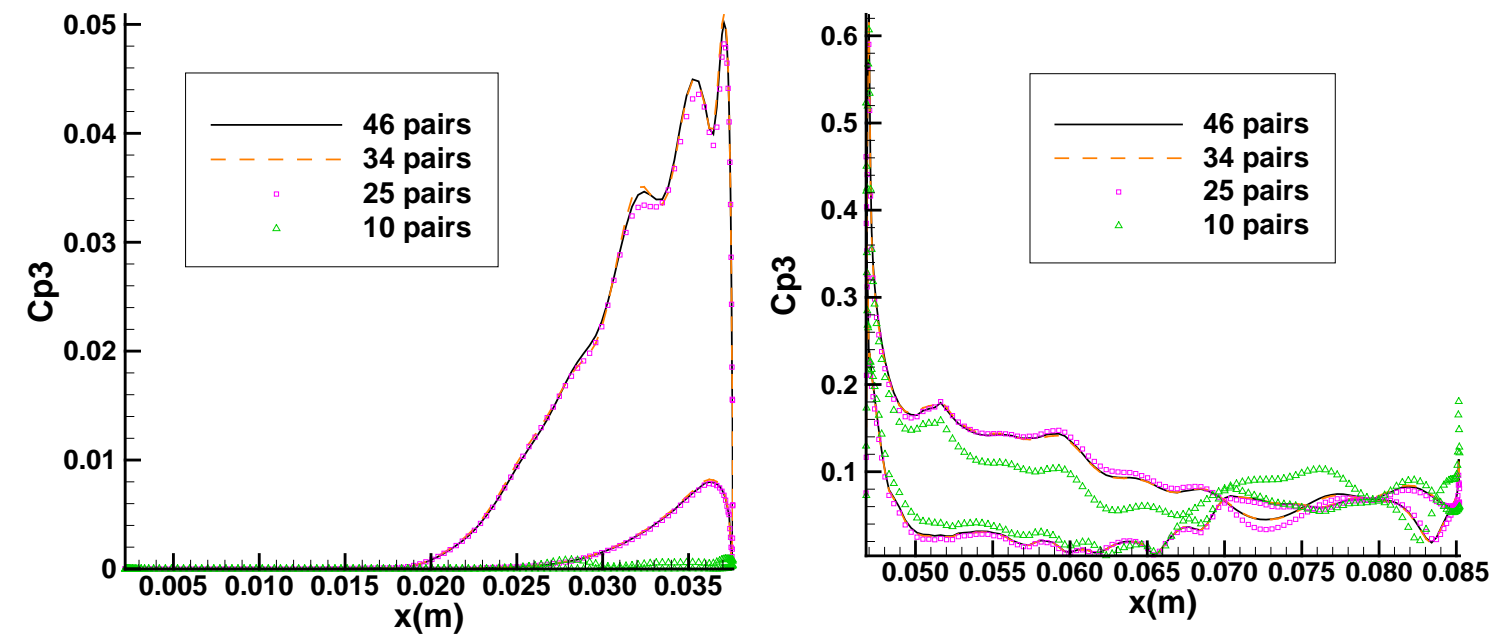

Figure 15 Comparison of amplitude of the third harmonic pressure coefficient stream-wise distribution at $50 \%$ span of the rotor (left) and the stator (right).
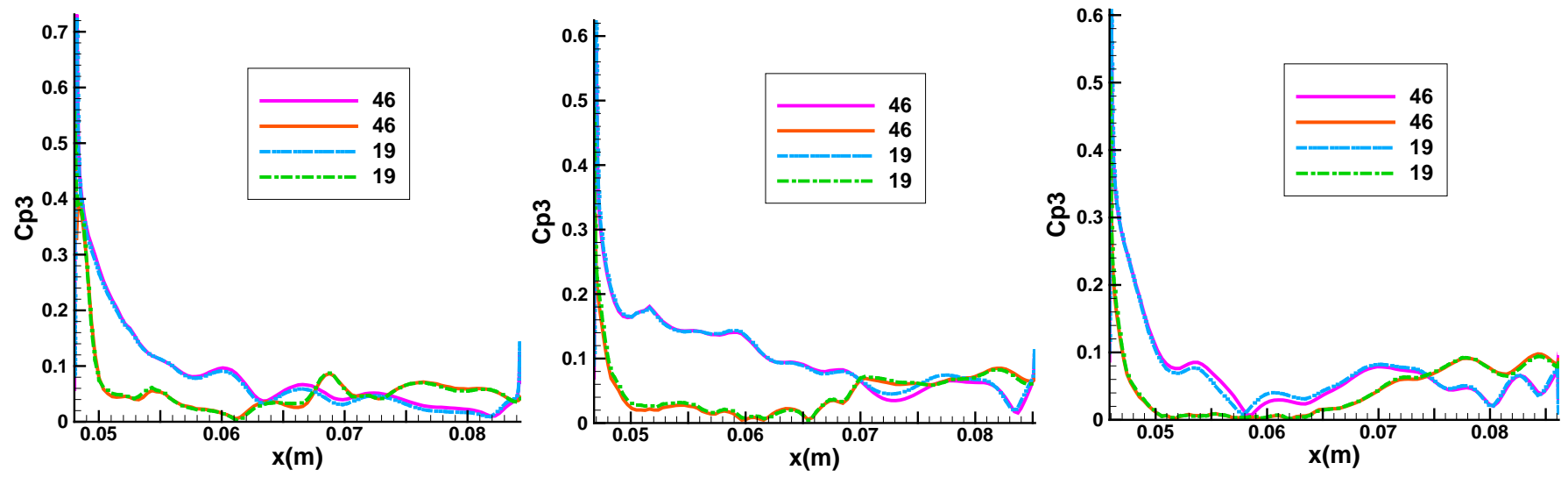

Figure 16 Comparison of amplitude of the third harmonic pressure coefficient stream-wise distribution at $10 \%$ span(left), $50 \%$ span(middle) and $90 \%$ span(right) of the stator domain .
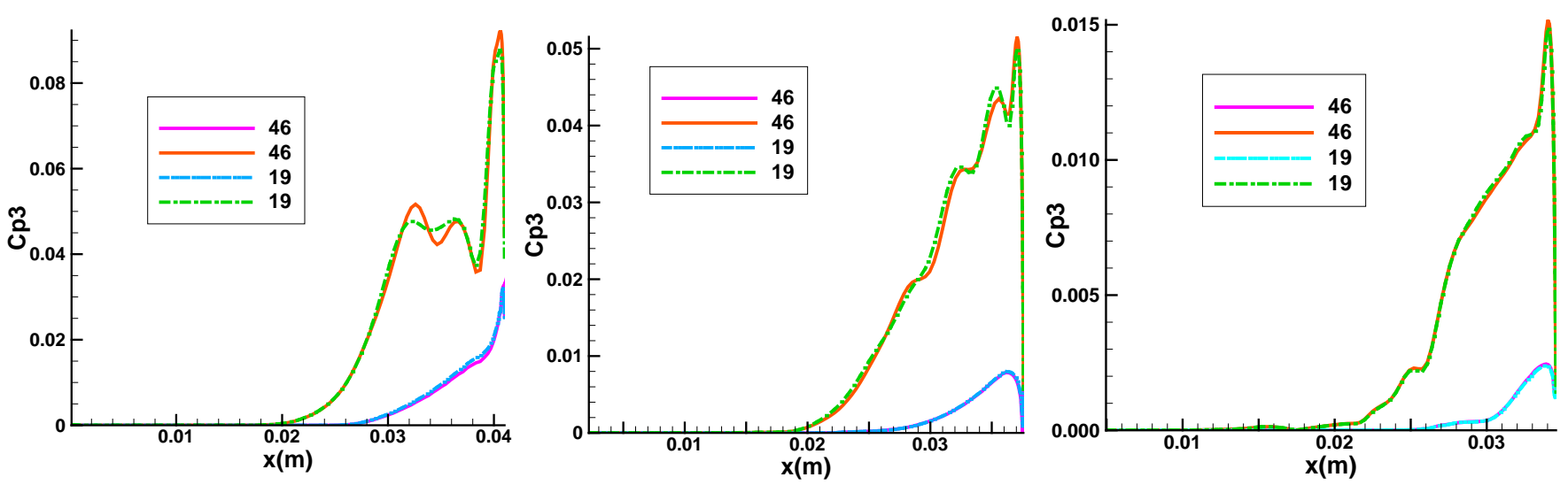

Figure 17 Comparison of amplitude of the third harmonic pressure coefficient stream-wise distribution at $10 \%$ span(left),50\% span(middle) and $90 \%$ span(right) of the rotor domain. 

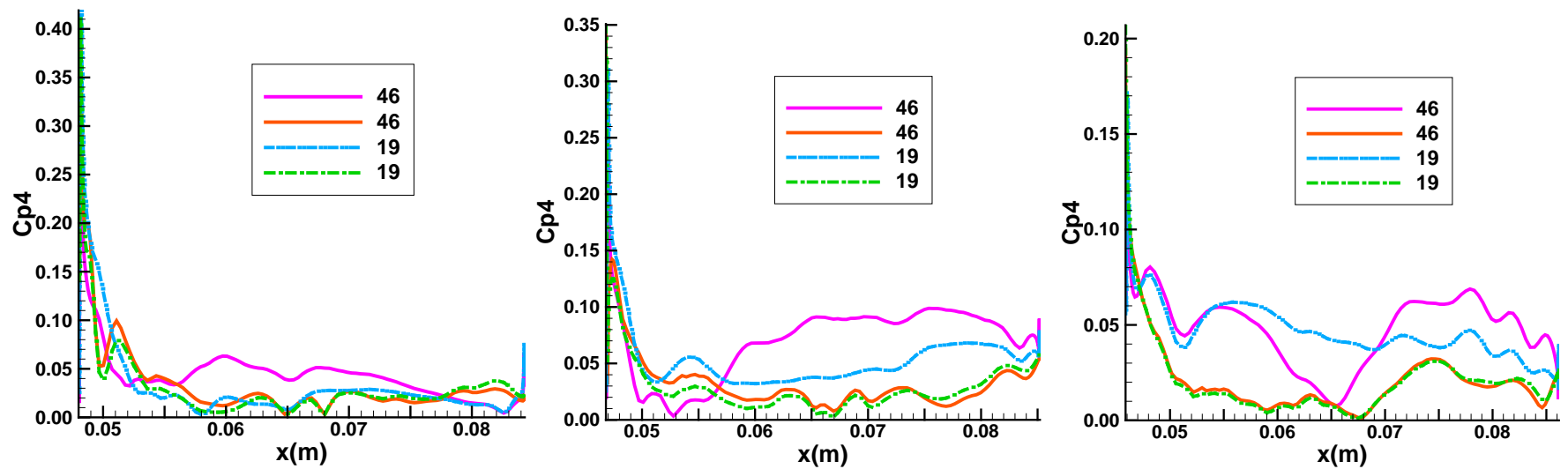

Figure 18 Comparison of amplitude of the fourth harmonic pressure coefficient stream-wise distribution at $10 \%$ span(left), $50 \%$ span(middle) and $90 \%$ span(right) of the stator domain.
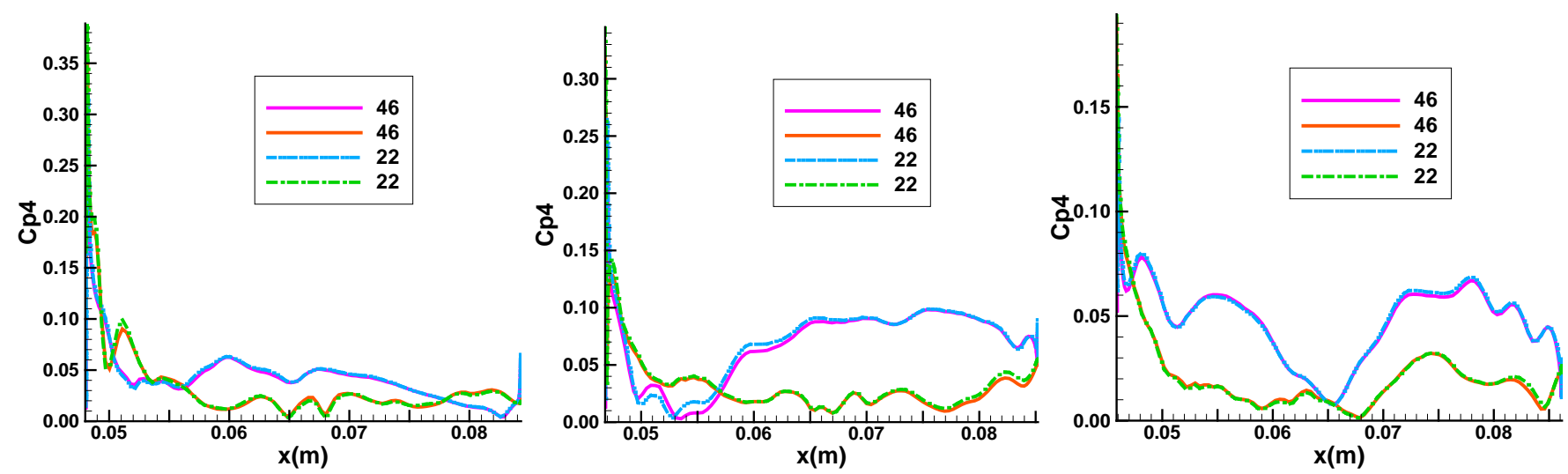

Figure 19 Comparison of amplitude of the fourth harmonic pressure coefficient stream-wise distribution at $10 \%$ span(left), $50 \%$ span(middle) and $90 \%$ span(right) of the stator domain .

Next, we need to pay more attention to the unsteady component of the flow field. This analysis is closely related to time and space mode decomposition and matching methods [15]. According to the above discussion, basic spinning modes, that is, the first three groups, are enough to approximate the overall performance and time averaged static pressure. While the unsteady component needs more spinning modes, which means the fourth and the fifth group need to contribute some spinning modes. The significant difference between the last two groups is that they have different nodal diameters. Apparently, the fourth group retains smaller nodal diameters, which tends to have more impacts on the results [20]. Based on this fact, a lot of numerical analyses were done.

From a lot of analyses, we find an interesting phenomenon. For example, if we focus on the amplitudes of the third harmonic pressure, part of the fourth group needs to be added on the basis of the first three groups. The fifth group can be totally dumped. And that part of the four group needs to satisfy the following expression

$\left(i \times N_{2} \times \omega,-i \times N_{2}+j \times N_{1}\right) \leftrightarrow\left(j \times N_{1} \times \omega,-i \times N_{2}+j \times N_{1}\right)$ where $i \in\left[1, n_{1}\right]$ and $j \in[1,3]$.

Hence there are $1+n_{1}+n_{2}+3 \times n_{1}$ harmonics in total to analyse the flow field, which is 19 in this case.

According to Figure 16 and Figure 17, the solution using 19 spinning modes compares well with the solution using the complete spinning modes on the amplitude of the third harmonic pressure. But the solution using 19 spinning modes does not agree with the solution using the complete spinning modes on the amplitude of the fourth harmonic pressure, shown in Figure 18. If the focus is set on the amplitude of the fourth harmonic pressure, then based on the above supplemented expression, the following part of fourth group is needed

$$
\left(i \times N_{2} \times \omega,-i \times N_{2}+j \times N_{1}\right) \leftrightarrow\left(j \times N_{1} \times \omega,-i \times N_{2}+j \times N_{1}\right)
$$

where $i \in\left[1, n_{1}\right]$ and $j \in[1,4]$.

There are $1+n_{1}+n_{2}+4 \times n_{1}$ harmonics in total to analyse the flow field, which is 22 in this case. The solution using 22 spinning modes is well consistent with the solution using the complete spinning modes (see Figure 19).

\section{CONCLUSIONS}

For the selection of the number of harmonics, discrete Fourier transform on conservative variables except $\rho w$ is performed at the exit of an upstream blade row and the inlet of a downstream blade row. Pick out the biggest number of harmonics at $10 \%, 50 \%, 90 \%$ three spans and average them. For the selection of spinning modes, if the overall performance and the time averaged static pressure is under consideration, using the first three groups in the time and 
space mode decomposition and matching method is enough to well resolve the flow field. If the amplitude of the harmonic pressure is a pressing matter, in addition to the first three groups, we need to add part of the fourth group. For example, if priorities are amplitude of the $\mathrm{M}$ harmonic pressure. Then the adding part is given by the following expression

$\left(i \times N_{2} \times \omega,-i \times N_{2}+j \times N_{1}\right) \leftrightarrow\left(j \times N_{1} \times \omega,-i \times N_{2}+j \times N_{1}\right)$

where $\mathrm{M}$ is the harmonic index, $i \in\left[1, n_{1}\right]$ and $j \in[1, M]$.

This study also demonstrates that good approximation of flow field can be achieved through a relatively small number of spinning modes.

\section{Nomenclature}

$E=$ total energy

$i, j=$ harmonics index

$N_{1} \quad=$ the number of blades in an upstream row

$N_{2} \quad=$ the number of blades in a downstream row

$n_{1}=$ the number of harmonics used in an upstream row

$n_{2} \quad=$ the number of harmonics used in a downstream row

$u=$ velocity component in the axial direction

$v=$ velocity component in the circumferential direction

$w \quad=\quad$ velocity component in the radial direction

$\lambda=$ the primitive turbulent solution variable of the Spalart Allmaras turbulence model equation

$\rho \quad=$ density

$\omega \quad=$ angular speed

\section{References}

[1] Hall, K. C., and C. B. Lorence. 1993. "Calculation of Three dimensional Unsteady Flows in Turbomachinery Using the Linearized Harmonic Euler Equations.” Journal of Turbomachinery 115 (4): 800-809.

[2] Hall, Kenneth C., and Edward F. Crawley. 1989. "Calculation of Unsteady Flows in Turbomachinery Using the Linearized Euler Equations.” AIAA Journal 27: 777-787.

[3] Clark, William S., and Kenneth C. Hall. 2000. "A Time linearized Navier-Stokes Analysis of Stall Flutter.” Journal of Turbomachinery 122: 467-476.

[4] He, L., and W. Ning. 1998. "An Efficient Approach for Analysis of Unsteady Viscous Flows in Turbomachines." AIAA Journal 36 (11): 2005-2012.

[5] Chen, T., P. Vasanthakumar, and L. He. 2001. "Analysis of Unsteady Blade Row Interaction Using Nonlinear Harmonic Approach.” Journal of Propulsion and Power 17 (3): 651-658. [6] Crespo, J., Corral, R., Pueblas, J., "An Implicit Harmonic Balance Method in Graphics Processing Units for Oscillating Blades" J. Turbomach 138(3), 031001.

[7] Ekici, Kivanc, Kenneth C. Hall, and Earl H. Dowell. 2008. "Computationally Fast Harmonic Balance Methods for Unsteady Aerodynamic Predictions of Helicopter Rotors." Journal of Turbomachinery 227: 6206-6225.

[8] Frey, C. , Ashcroft, G. , Kersken, H. , and Voigt, C. , 2014, "A Harmonic Balance Technique for Multistage Turbomachinery Applications,” ASME Paper No. GT201425230.

[9] Hall K C, Ekici K, Thomas J P, et al. Harmonic balance methods applied to computational fluid dynamics
problems[J]. International Journal of Computational Fluid Dynamics, 2013, 27(2):52-67.

[10] Hall K C., J. P. Thomas, and W. S. Clark. 2002. "Computation of Unsteady Nonlinear Flows in Cascades Using a Harmonic Balance Technique.” AIAA Journal 40 (5): 879-886.

[11] Sicot, Frédéric, Guédeney, Thomas, Dufour G. Timedomain harmonic balance method for aerodynamic and aeroelastic simulations of turbomachinery flows[J]. International Journal of Computational Fluid Dynamics, 2013, 27(2):68-78.

[12] van derWeide, Edwin, Arathi K. Gopinath, and Antony Jameson. 2005. "Turbomachinery Applications with the Time Spectral Method.” AIAA Paper 2005 - 4905.

[13] Maple R C, King P I, Orkwis P D, et al. Adaptive harmonic balance method for nonlinear time-periodic flows[J]. Journal of Computational Physics, 2004, 193(2):620-641.

[14] Gomar, Adrien, Quentin Bouvy, Frédéic Sicot, Paola Dufour Guillaume, Cinnella, and Benjamin François. 2014. "Convergence of Fourier-based Time Methods for Turbomachinery Wake Passing Problems.” Journal of Computational Physics 278: 229-256.

[15] Dingxi W, Xiuquan H. A complete rotor-stator coupling method for frequency domain analysis of turbomachinery unsteady flow[J]. Aerospace Science and Technology, 2017, 70:367-377.

[16] Xiuquan H, Hangkong W, Dingxi W. Implicit solution of harmonic balance equation system using the LU-SGS method and one-step Jacobi/Gauss-Seidel iteration[J]. International Journal of Computational Fluid Dynamics, 2018:1-15.

[17] Tyler, J. M., and Sofrin, T. G., 1962. "Axial Flow Compressor Noise Studies”. SAE Transaction, Vol. 70, pp. 309-332.

[18] Spalart P, Allmaras S. A one-equation turbulence model for aerodynamic flows[J]. Recherche Aerospatiale, 1994, 1(1):5-21.

[19] Reid, Lonnie, and Royce D. Moore. 1978. "Performance of Single-stage Axial-flow Transonic Compressor with Rotor and Stator Aspect Ratios of 1.19 and 1.26, Respectively, and with Design Pressure Ratio of 1.82.” NASA TP 1338.

[20] Hall K C. Multistage Coupling for Unsteady Flows in Turbomachinery[J]. Unsteady Aerodynamics, Aeroacoustics and Aeroelasticity of Turbomachines, 2006. 